\title{
Clinical and serological prognostic markers in primary biliary cholangitis
}

\author{
Markus Wilhelmi \\ Department of Gastroenterology, Hepatology, and Nutritional Medicine (Medicine Clinic I), University Hospital Frankfurt, Frankfurt am \\ Main, Germany
}

Keywords: Primary biliary cholangitis; Prognosis; Antibodies, Antinuclear; Anti-gp-210 antibodies; Cirrhosis

Dear Editor,

We read with great interest the review article by Tanaka, aiming to analyze the currently well-defined epidemiological, etiological, genetic, clinical-diagnostic, and finally therapeutic aspects of primary biliary cholangitis (PBC). Although we find the review very comprehensive, we would like to add a comment that we consider relevant on some prognostic markers of PBC, not mentioned by Tanaka. In particular, when the author discusses clinical and serological aspects, in our opinion, he neglects to mention the prognostic significance of specific autoantibodies as well as of some symptoms at the clinical presentation of the disease. From the clinical point of view, we think it is worth mentioning that the presence of fatigue and or pruritus at the onset of the disease is associated with a disease that is particularly active, less responsive to treatment with ursodeoxycholic acid treatment, and more inclined to evolve to cirrhosis and its complications. ${ }^{2,3}$ Also of great relevance is the recognized prognostic role of some anti-nuclear autoantibodies. In particular, anti-gp210 antibody positivity is correlated with poor outcomes and many disease progression types, especially liver failure. Therefore, this marker allows identifying the subgroup of PBC patients who deserve close follow-up to assess the need for second-line treatment. ${ }^{4}$ Finally, from a diagnostic point of view, it is also widely established the very high diagnostic accuracy of some anti-nuclear antibodies in patients who lack anti-mitochondrial antibodies, as demonstrated by several previous studies. ${ }^{5-10}$ In particular, it has been reported that concomitant positivity for both anti-Sp100 and anti-gp210 antibodies showed a $100 \%$ positive predictive value for PBC, irrespective of the antimitochondrial antibody status. ${ }^{11}$

\section{Conflicts of Interest}

The author has no conflicts of interest to disclose.

\section{REFERENCES}

1. Tanaka A. Current understanding of primary biliary cholangitis. Clin Mol Hepatol 2021;27:1-21.

2. Quarneti C, Muratori P, Lalanne C, Fabbri A, Menichella R, Granito A,

\section{Abbreviation:}

PBC, primary biliary cholangitis

\section{Corresponding author: Markus Wilhelmi}

Department of Gastroenterology, Hepatology, and Nutritional Medicine (Medicine Clinic I), University Hospital Frankfurt, Theodor-Stern-Kai 7, Frankfurt am Main 60590, Germany

Tel: +49 69 63010, Fax: +4969 6301-81671

E-mail: dr.markus.wilhelmi@gmail.com

https://orcid.org/0000-0003-0121-0627 
et al. Fatigue and pruritus at onset identify a more aggressive subset of primary biliary cirrhosis. Liver Int 2015;35:636-641.

3. Carbone M, Mells GF, Pells G, Dawwas MF, Newton JL, Heneghan $M A$, et al. Sex and age are determinants of the clinical phenotype of primary biliary cirrhosis and response to ursodeoxycholic acid. Gastroenterology 2013;144:560-569.e7; quiz e13-e14.

4. Huang C, Han W, Wang C, Liu Y, Chen Y, Duan Z. Early prognostic utility of gp210 antibody-positive rate in primary biliary cholangitis: a meta-analysis. Dis Markers 2019;2019:9121207.

5. Granito A, Muratori P, Quarneti C, Pappas G, Cicola R, Muratori L. Antinuclear antibodies as ancillary markers in primary biliary cirrhosis. Expert Rev Mol Diagn 2012;12:65-74.

6. Muratori P, Granito A, Ferri S, Pappas G, Volta U, Menichella R, et al. Multiple nuclear dots and rim-like/membranous IgG isotypes in primary biliary cirrhosis. Autoimmunity 2009;42:224-227.

7. Granito A, Yang WH, Muratori L, Lim MJ, Nakajima A, Ferri S, et al.
PML nuclear body component Sp140 is a novel autoantigen in primary biliary cirrhosis. Am J Gastroenterol 2010;105:125-131.

8. Muratori P, Granito A, Pappas G, Muratori L, Lenzi M, Bianchi FB. Autoimmune liver disease 2007. Mol Aspects Med 2008;29:96-102.

9. Granito A, Muratori L, Tovoli F, Muratori P. Surrogate markers for antimitochondrial antibody-negative primary biliary cholangitis. Am J Gastroenterol 2021;116:215-217.

10. Granito A, Muratori P, Muratori L, Pappas G, Cassani F, Worthington $J$, et al. Antibodies to SS-A/Ro-52kD and centromere in autoimmune liver disease: a clue to diagnosis and prognosis of primary biliary cirrhosis. Aliment Pharmacol Ther 2007;26:831-838.

11. Granito A, Muratori P, Muratori L, Pappas G, Cassani F, Worthington $J$, et al. Antinuclear antibodies giving the 'multiple nuclear dots' or the 'rim-like/membranous' patterns: diagnostic accuracy for primary biliary cirrhosis. Aliment Pharmacol Ther 2006;24:1575-1583. 